\title{
Peculiarities of Excitonic Photoluminescence in Si $\delta$-Doped GaAs Structures
}

\author{
V. NArgeliente ${ }^{a}$, S. Ašmontas ${ }^{a}$, A. ČeršKus ${ }^{a, b}$, J. Gradauskas ${ }^{a, c}$, \\ J. KundROTAS ${ }^{a, c, *}$ AND A. SuŽIEDĖLIS ${ }^{a, c}$ \\ ${ }^{a}$ Semiconductor Physics Institute, Center for Physical Sciences and Technology \\ A. Goštauto 11, LT-01108 Vilnius, Lithuania \\ ${ }^{b}$ Department of General Physics, Vilnius Pedagogical University, Studentu̧ 39, LT-08106 Vilnius, Lithuania \\ ${ }^{c}$ Vilnius Gediminas Technical University, Saulètekio 11, LT-10223 Vilnius, Lithuania \\ We present investigation of photoluminescence properties of $\mathrm{Si} \delta$-doped GaAs structures at different \\ temperatures and various laser excitation intensities. Strong excitonic emission was observed in the $\delta$-doped \\ structures. The photoluminescence in the infrared region, below excitonic emission, originates from a non-phonon \\ free electron-acceptor $\mathrm{e}-\mathrm{A}$ transitions and longitudinal optical phonon sidebands of $\mathrm{e}-\mathrm{A}$ transitions. Possible \\ mechanisms for recombination of photocarriers are discussed, with a particular focus on an enhanced excitonic \\ photoluminescence emission in comparison with that from intrinsic GaAs layers of the same structures.
}

PACS: 78.55.-m, 71.35.-y, 68.65.-k

\section{Introduction}

Since its first suggestion, $\delta$-doping has attracted considerable attention due to unique electronic properties of the $\delta$-doped structures that are extremely useful to study quantum confinement of charge carriers [1]. The $\delta$-doped structures are usually characterized by a rather high carrier density, which makes them unique among the other two-dimensional (2D) systems. Specific properties of the $\delta$-doped systems make them interesting for both fundamental point of view and application in design and fabrication of electronic devices such as HEMTs [2], $\delta$-FETs $[3,4]$, tunneling diodes $[5,6]$.

In this report, we present investigation of photoluminescence (PL) properties of $\delta$-doped GaAs structures and compare their PL spectrum with that of intrinsic GaAs layers of the same structures.

\section{Samples growth and experimental technique}

The investigated silicon $\delta$-doped GaAs samples were grown using the molecular beam epitaxy technique. As a buffer layer undoped GaAs layer with thickness of 1 or $0.9 \mu \mathrm{m}$ and $20 \mathrm{Al}_{x} \mathrm{Ga}_{1-x} \mathrm{As} / \mathrm{GaAs}$ multiple quantum well layers were grown on semi-insulating GaAs substrates. The parameters of the $\mathrm{Al}_{x} \mathrm{Ga}_{1-x} \mathrm{As} / \mathrm{GaAs}$ multiple quantum wells were different for the samples \#1 and \#2: well width $L_{\mathrm{W}}=6 \mathrm{~nm}$ and barrier thickness $L_{\mathrm{B}}=31 \mathrm{~nm}$ with composition $x=0.36$ in the case of the sample $\# 1$, and $L_{\mathrm{W}}=5 \mathrm{~nm}, L_{\mathrm{B}}=30 \mathrm{~nm}, x=0.3$ in the case of the sample \#2. After that, a $50 \mathrm{~nm}$ undoped GaAs layer and a $\mathrm{Si} \delta$-doped layer were grown. The sample \#1 contained single $\delta$-doped layer with $\mathrm{Si}$ atoms concentration

* corresponding author; e-mail: kundrot@pfi.lt of $5 \times 10^{17} \mathrm{~cm}^{-3}$ and thickness of the $\delta$ layer $20 \mathrm{~nm}$. The sample \#2 contained fivefold $\delta$-doped layers separated by $25 \mathrm{~nm}$ undoped $i$-GaAs. For the multilayered structure the thickness of each $\delta$-doped layer was $5 \mathrm{~nm}$ and $\mathrm{Si}$ atoms concentration was $2 \times 10^{18} \mathrm{~cm}^{-3}$. Both samples were capped with undoped GaAs layer of $50 \mathrm{~nm}$ thickness.

The photoluminescence was excited by a continuous wave argon-ion laser with photon energy $2.4-2.7 \mathrm{eV}$ or $\mathrm{HeCd}(3.8 \mathrm{eV})$ laser. The PL signal was then dispersed by a monochromator and detected by a thermoelectrically cooled GaAs photomultiplier operating in a photon counting regime. We used closed cycle helium cryostat to achieve various cryogenic temperatures down to $3.6 \mathrm{~K}$.

For investigation of intrinsic GaAs layers and observation of changes in PL spectra in the $\delta$-doped structures the $\delta$-doped layer was removed. A chemical etching using $\mathrm{H}_{3} \mathrm{PO}_{4}: \mathrm{H}_{2} \mathrm{O}_{2}: \mathrm{H}_{2} \mathrm{O}=1: 1: 50$ was performed.

\section{Results and discussion}

The PL spectra of the $\delta$-doped GaAs samples close to liquid helium temperature are shown in Fig. 1. One can resolve two parts of the spectra. The first one, above $1.58 \mathrm{eV}$, we relate with the multiple quantum wells in the buffer layer, and the second one, below GaAs forbidden energy gap $E_{\mathrm{g}}(\mathrm{GaAs})=1.519 \mathrm{eV}$, we associate with the GaAs layer. The lower energy transitions at $1.49 \mathrm{eV}$, labeled $\mathrm{e}-\mathrm{A}$, are related to the recombination of free electron with residual acceptor in GaAs. The line marked as $X$ originates from emission of free excitons (bound excitons are not clearly distinguished in Fig. 1). Meanwhile, a ratio between the excitonic line and $\mathrm{e}-\mathrm{A}$ line $\mathrm{PL}$ intensities is about five times higher in the sample with fivefold $\delta$ layer than that in the sample with a single $\delta$ layer. Excitonic line with five doped layers is about 2.5 
times more intensive than the excitonic line of the single $\delta$-doped sample. The excitonic line full width at half maximum is about $4 \mathrm{meV}$ for both samples.

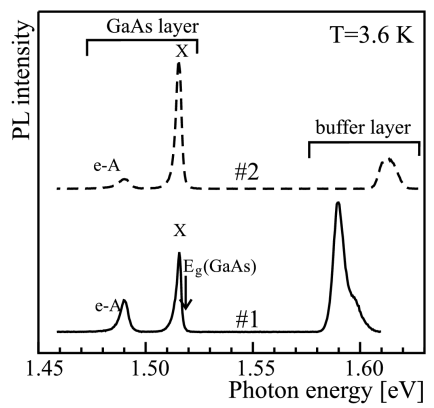

Fig. 1. The PL spectra of Si $\delta$-doped GaAs samples measured at $T=3.6 \mathrm{~K}$ temperature. $\mathrm{X}$ indicates excitonic transition and label e-A marks PL associated with residual acceptor.

To gain a deeper insight into the PL features, the PL spectra have been studied as a function of laser excitation intensity (Fig. 2) and lattice temperature (Fig. 3). Detailed study of the PL experimental results shows appearance of two new lines of low intensity on the lower energy side of the spectra. They are designated as e- $\mathrm{A}_{1 \mathrm{LO}}$ and $\mathrm{e}-\mathrm{A}_{2 \mathrm{LO}}$, which corresponds to phonon replicas of e-A transitions. These phonon satellites are associated with longitudinal optical (LO) phonon with energy $\hbar \omega_{\mathrm{LO}}=36.75 \mathrm{meV}[7]$.

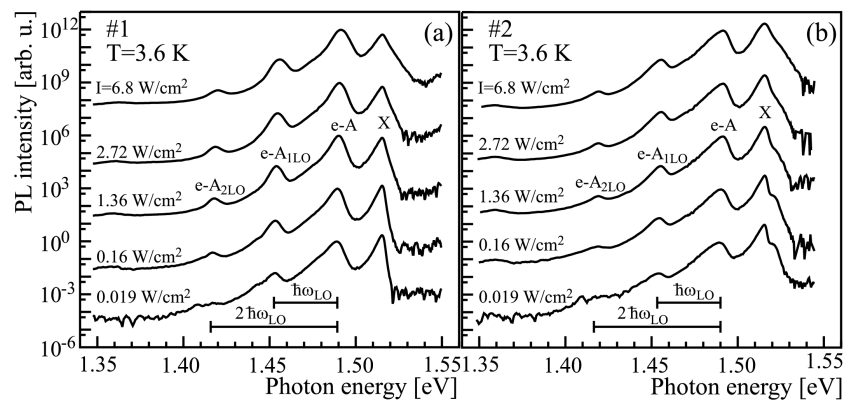

Fig. 2. The PL spectra of Si $\delta$-doped GaAs samples with (a) single and (b) fivefold $\delta$ layer measured at $T=$ $3.6 \mathrm{~K}$ and at different Ar ion laser excitation intensities.

From the sample $\# 1$ measurements at various laser excitation intensities, as seen from Fig. 2a, we clearly observe that $\mathrm{PL}$ intensity of $\mathrm{e}-\mathrm{A}$ line grows with the increase of the laser excitation. The measurements at different temperatures show that $\mathrm{e}-\mathrm{A}$ line saturates and vanishes at $40 \mathrm{~K}$ temperature (Fig. 3a). The distances between all lines are independent of temperature and laser excitation intensity. These features are typical for impurity related photoluminescence. Similar properties of the spectra were obtained for the sample \#2 with five $\delta$ layers (Figs. 2b, 3b).

We investigated PL depending on the penetration of laser light into the structures using argon ion or $\mathrm{HeCd}$

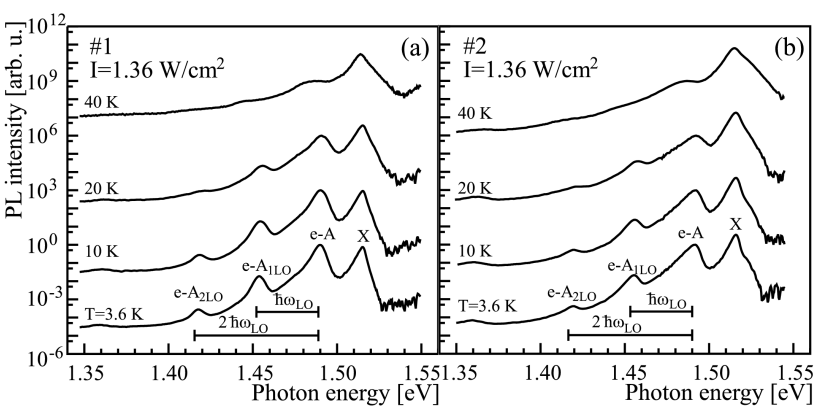

Fig. 3. The PL spectra of Si $\delta$-doped GaAs samples with (a) single and (b) fivefold $\delta$ layer at different lattice temperatures.

lasers. No substantial difference in the PL spectra was observed. However, great difference exists in the PL spectra of $i$-GaAs layer and the delta doped structure. The PL spectra of the single $\delta$-doped GaAs sample $(\# 1)$ before and after etching of the $\delta$-doped layer are compared in Fig. 4. It can be seen that excitonic lines are enhanced in the $\delta$-doped sample in comparison with the PL intensity of the intrinsic GaAs. The excitonic line of the $\delta$-doped sample is about 500 times more intensive than that in GaAs after removal of the $\delta$ layer.

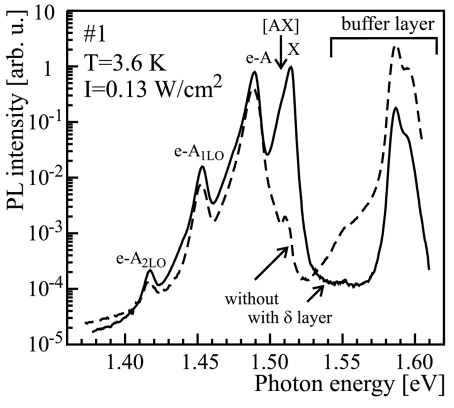

Fig. 4. The PL spectra of $\mathrm{Si} \delta$-doped GaAs samples (\#1) before (solid line) and after etching of $\delta$ layer (dashed line). [AX] indicates acceptor bound exciton. $\mathrm{HeCd}$ laser was used as an excitation source.

From the intensities of PL replicas, the Huang-Rhys factor $S=2 I_{2 \mathrm{LO}} / I_{1 \mathrm{LO}}$, where $I_{1 \mathrm{LO}}$ is intensity of the first and $I_{2 \mathrm{LO}}$ is intensity of the second phonon replica of e-A transition [8], respectively, was estimated. The obtained $S$ value for the sample with a single $\delta$-doped layer was equal to $0.03-0.034$, for the sample with five $\delta$ layers it was equal to 0.03-0.039, and for the etched GaAs structure it equaled 0.04, which is in agreement with the value $S=0.05$ for acceptor doped GaAs epilayer [8].

Before starting discussion on excitonic emission we solved self-consistent Schrödinger-Poisson equations [9]. Energy band diagram, electron concentration and electric field strength in the $\delta$-doped GaAs samples were calculated. The calculations were made at $T=3.6 \mathrm{~K}$ temperature assuming that donors are fully ionized. The calculated results are shown in Fig. 5. A surface poten- 
tial $\Phi_{\mathrm{S}}=0.6 \mathrm{eV}$ was taken into account, which leads to asymmetric energy band diagrams at the surface and to lowering of free carriers concentration about three times in the doping notch of the single $\delta$ doped layer. The surface potential influences only the first doping notch of the fivefold $\delta$ doped layer.

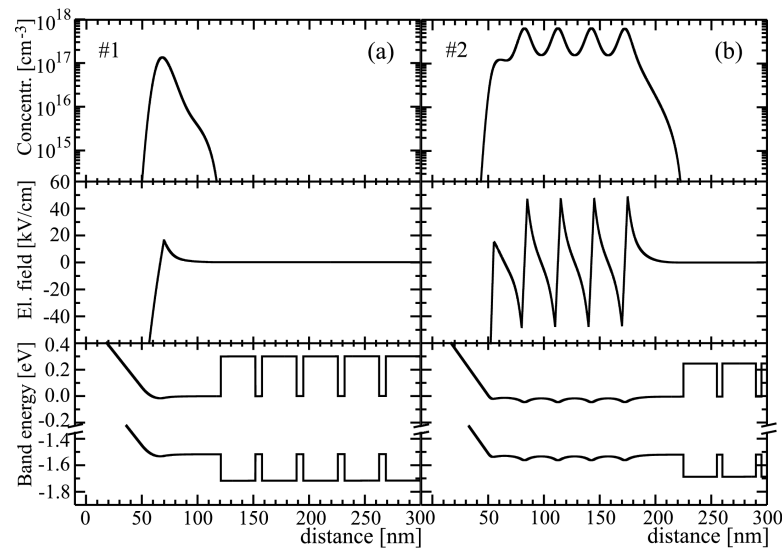

Fig. 5. Numerical calculations of the energy band diagram (bottom), built-in electric field (middle) and electron concentration (top) of (a) single and (b) fivefold $\delta$-doped GaAs with surface potential $\Phi_{\mathrm{S}}=0.6 \mathrm{eV}$.

The reasons of the origin of strong excitonic PL in the $\delta$-doped structures with $2 \mathrm{DEG}$ is not obvious. Calculations indicate that the $\delta$-doped structures induce electric field. For example, the effect of built-in electric field was used to explain the origin of the excitonic photoluminescence in selectively doped $\mathrm{Al}_{x} \mathrm{Ga}_{1-x} \mathrm{As} / \mathrm{GaAs}$ heterojunctions $[10,11]$.

The incident laser beam excites electrons to a certain light penetration depth. These photoexcited carriers can form excitons in the GaAs structure and can recombine back by emitting photons. However, the presence of a strong built-in electric field governs exciton formation in the close proximity of the $\delta$-doped region.

In the doped region, 2DEG forms the quasi V-shaped potential well and induces an electric field of the order of $20 \mathrm{kV} / \mathrm{cm}$ (see Fig. 5). However, it is well known that an electric field above $1 \mathrm{kV} / \mathrm{cm}$ can destroy excitons by tunneling [12]. Therefore, the electron-hole pairs near the $\delta$ region are dissolved by the electric field and will either move towards (electrons) or away (holes) from the $\delta$ region. During this process the dissolved electron and hole cannot meet each other, but may have the possibility to catch holes and electrons originating from the other pairs in a bimolecular exciton formation. The resulting process can lead to accumulation of free carriers thus increasing the number of excitons in the GaAs flat band region. If the recombination processes are influenced by the impurities, the excitonic PL intensity depends on ex- citation intensity or on concentration of carriers forming excitons according to the square law [13]. This nonlinear behavior of excitonic PL intensity can help explain the enhancement of excitonic lines.

\section{Summary}

In $\mathrm{PL}$ spectra of $\mathrm{Si} \delta$-doped GaAs structures we observe strong excitonic emission in comparison with the same samples but having etched-out $\delta$-doped layer. Electron-acceptor transitions and its first and second longitudinal optical phonon replicas in both $\delta$-doped and etched $\delta$-doped layer structures dominate in the emission below the excitonic line. We suppose that enhanced excitonic emission in the $\delta$-doped structures is related with the induced electric field near the doping notch which leads to a nonlinear behaviour of excitonic photoluminescence.

\section{Acknowledgments}

This work was supported by Research Council of Lithuania under the contract TAP-56/2010. The MBE grown structures were fabricated at Braun Center for Submicron Research under the contract No. RITA-CT-2003-506095. The authors acknowledge A. Steikūnienè and G. Steikūnas for preparing of the samples.

\section{References}

[1] Delta-Doping of Semiconductors, Ed. E.F. Schubert, Cambridge University Press, Cambrige 1996.

[2] M.K. Tsai, S.W. Tan, Y.W. Wu, W.S. Lour, Y.J. Yang, Semicond. Sci. Technol. 17, 156 (2002).

[3] E.F. Schubert, K. Ploog, Jpn. J. Appl. Phys. 24, L608 (1985).

[4] J.C. Martínez-Orozco, I. Rodríguez-Vargas, M.E. Mora-Ramos, C.A. Duque, Microelectron. J. 39, 648 (2008).

[5] J.M.A. Gilman, A.G.O. O'Neill, Electron. Lett. 26, 601 (1990).

[6] S.A. Vitusevich, A. Förster, A.E. Belyaev, B.A. Glavin, K.M. Indlekofer, H. Lüth, R.V. Konakova, Semicond. Phys., Quant. Electron. Optoelectron. 2, 7 (1999).

[7] A. Mooradian, G.B. Wright, Solid State Commun. 4 , 431 (1966).

[8] J. Kundrotas, A. Čerškus, S. Ašmontas, G. Valušis, M.P. Halsall, E. Johannessen, P. Harrison, Semicond. Sci. Technol. 22, 1070 (2007).

[9] I.-H. Tan, G.L. Snider, L.D. Chang, E.L. Hu, J. Appl. Phys. 68, 4071 (1990).

[10] J.X. Shen, R. Pittini, Y. Oka, Phys. Rev. B 64 195321 (2001).

[11] B.M. Ashkinadze, E. Linder, V. Umansky, Phys. Rev. B 62, 10310 (2000)

[12] L. Schultheis, K. Köhler, C.W. Tu, Phys. Rev. B 36 6609 (1987).

[13] T. Schmidt, K. Lischka, W. Zulehner, Phys. Rev. B 45, 8989 (1992). 